\title{
Designing of Adaptive Model-Free Controller Based on Output Error and Feedback Linearization
}

\author{
S. Pezeshki, ${ }^{1}$ M. A. Badamchizadeh, ${ }^{1}$ S. Ghaemi, ${ }^{1}$ and M. A. Poor ${ }^{2}$ \\ ${ }^{1}$ Control Engineering Department, Faculty of Electrical and Computer Engineering, University of Tabriz, Tabriz 5166614776, Iran \\ ${ }^{2}$ Department of Mechanical and Industrial Engineering, Concordia University, Montreal, QC, Canada
}

Correspondence should be addressed to M. A. Badamchizadeh; mbadamchi@tabrizu.ac.ir

Received 20 September 2012; Accepted 30 December 2012

Academic Editor: Shukai Duan

Copyright (C) 2013 S. Pezeshki et al. This is an open access article distributed under the Creative Commons Attribution License, which permits unrestricted use, distribution, and reproduction in any medium, provided the original work is properly cited.

\begin{abstract}
This paper introduces a new approach to design Model-Free Adaptive Controller (MFAC) using adaptive fuzzy procedure as a feedback linearization based on output error. The basic idea is to transfer the control signal to an appropriate surface and then, depending on the output error of system, the control signal changes around this surface. Some examples are provided as well to illustrate the efficiency of the proposed approach. The obtained simulation results have shown good performances of the proposed controller.
\end{abstract}

\section{Introduction}

Classical control method is based on mathematical equations of the system; however, this method suffers from some drawbacks. For example, in this method the performance of system can be affected by unmodeled dynamics of system and/or by large delays. Model-Free Adaptive Control (MFAC), as a part of modern control theory, shows superiorities compared to model-based methods. MFAC is an adaptive control method and needs no information about system model. It only uses $I / O$ data for controller design and therefore it is a nonlinear controller designed without the need to mathematical model of controlled system [1-3].

In 1994, Han and Wang introduced model-free topic and proved the stability of MFAC controllers. In 1993-1994, Hou Zhongsheng represented applications of these controllers. With using pseudo-Jacobian matrix, nonlinear systems are replaced to near the rail line of controlled system and then the $I / O$ data of controlled system are used to design MFAC controller [4].

In 1995, Jagannathan suggested a fuzzy stable controller for a limited class of nonlinear system in the form of $x(k+$ $1)=f(x(k))+u(k)$, where $f(x)$ is the unknown nonlinear function. In 1996, this controller was used for general class of nonlinear system in the form of $x(k+1)=f(x(k))+$ $g(x(k)) u(k)[5]$.
Another idea is introduced in 2000 [6], which uses a neural network as an adaptive controller for stabilization of system. Their proposed algorithm does not need any complex tuning and can be applied to any controllable multiinput systems.

Compared with other adaptive control schemes, the MFAC approach has several advantages, which make this method suitable for control applications. First, MFAC just depends on the real-time measurement data of the controlled plant. Second, MFAC does not require any other testing signals and any training process. Third, MFAC is simple and easily implementable with small computational burden and has strong robustness. Fourth, MFAC approach does not need a specific controller for each specific process. Finally, the MFAC has been successfully implemented in many practical applications, for example, chemical industry, linear motor control, injection modeling process, $\mathrm{PH}$ value control, and so on [4].

The main contribution of this work is to introduce a new MFAC approach based on output error and feedback linearization. The proposed model has rapid monotonic tracking error convergence, robust stability, and good disturbance rejection.

The rest of the paper is organized as follows: in Section 2, adaptive fuzzy control based on feedback linearization is 
described. The proposed model-free adaptive controller and its Lyapunov stability are represented in Sections 3 and 4, respectively; in Section 5 simulation results are given to highlight advantages of MFAC method. Finally conclusion is stated in Section 6.

\section{Feedback Linearization by Means of Adaptive Fuzzy Controller}

In feedback linearization method, control law is determined in such a way that nonlinear terms eliminate the system dynamic and replace it with appropriate reference input as seen below: (for simplifying $x(t)=x$ ),

$$
\begin{aligned}
\dot{x}(t) & =f(x)+g(x) u(t) \longrightarrow u(t) \\
& =g^{-1}(x)(-f(x)+v(t)) .
\end{aligned}
$$

Conceptually, Adaptive Feedback Linearization (AFL) method is the same as nonadaptive counterpart but AFL uses adaptation law for updating controller parameter to enhance system performance. Notice that both methods should have complete description of system dynamic but our proposed method does not need any information of system dynamic. Due to the fact that it is difficult to obtain a mathematical model of the system and typically there exist some numerical approximations, the object of this paper is to control systems just with $I / O$ data in order to improve system performance. In order to reach this goal it is required to estimate unknown nonlinear function of system dynamic $f(x)$ and $g(x)$ with $\hat{f}(x)$ and $\hat{g}(x)$ or estimate control law $u(t)$ with $\widehat{u}(x)$. Estimation algorithm in this approach utilizes basic fuzzy membership function. As an instance for estimating $f(x)$, we have

$$
f(x) \longrightarrow \widehat{f}(x)=\frac{\sum_{i=0}^{p} c_{i} \mu_{i}}{\sum_{i=0}^{p} \mu_{i}},
$$

where $p$ is the number of fuzzy roles, $\mu_{i}(x)$ is membership function for $i$ th fuzzy role, also $c_{i}$ is and the result of $i$ th fuzzy role for system that is generally considered as linear combination of some series of continuous functions such as $z(x)$ :

$$
\begin{aligned}
& z_{k}(x) \in R, \quad k=1,2, \ldots, m-1, \\
& c_{i}=a_{i, 0}+a_{i, i} z_{1}(x)+\cdots+a_{i, m-1} z_{m-1}(x) \text {, } \\
& z=\left[\begin{array}{llllll}
1 & z_{1}(x) & z_{2}(x) & \cdots & z_{m-1}(x)
\end{array}\right]^{T}, \\
& A^{T}=\left[\begin{array}{cccc}
a_{1,0} & a_{1,1} & \cdots & a_{1, m-1} \\
\vdots & & & \vdots \\
a_{p, 0} & a_{p, 1} & \cdots & a_{p, m-1}
\end{array}\right] \text {, } \\
& c=z^{T} A
\end{aligned}
$$

Using (3), (4), $\widehat{f}(x)$ can be rewritten as

$$
\begin{gathered}
\xi=\frac{\left[\begin{array}{llll}
\mu_{1} & \mu_{2} & \cdots & \mu_{p}
\end{array}\right]}{\sum_{i=0}^{p} \mu_{i}}, \\
f(x)=z^{T} \cdot A \cdot \zeta+\varepsilon(x) \Longrightarrow \widehat{f}(x)=z^{T} \cdot A \cdot \zeta,
\end{gathered}
$$

where $\varepsilon(x)$ is the approximation error of fuzzy approach. $A(t)$ is updated by the adaptation law below:

$$
\dot{A}(t)=-Q^{-1} \cdot z \cdot \xi^{T} \cdot \text { es. }
$$

$Q$ is a constant coefficient that usually is selected small. es is the difference between output $x$ and reference output $x_{\text {ref }}$ :

$$
\text { es }=x-x_{\text {ref }} \text {. }
$$

It should be pointed that AFC guarantees that $A(t)$ approaches to its desired value, $A^{*}(t)$.

Notice that the initial values of $A(t)$ can be chosen as $A(0)=0$ or any selected value by information that is acquired from system dynamic or even can be determined from another available control approach; for instance, the columns of $A(0)$ can be taken as $k_{f}\left(k_{f}\right.$ is obtained from state feedback method $u=-k_{f} x$ ) or, for example, if we want to estimate $f(x)$ function, the columns of $A(0)$ are better to contain coefficients of system states of $f(x)$ [7]. Fuzzy rules are expressed as follows:

$$
\text { If } X \text { is } F_{i} \text { then } c_{i}=f_{i}(z) \text {. }
$$

$f_{i}(z)$ is related to $i$ th row of $z^{T} A$ matrix. So the unknown nonlinear function $f(x)$ is estimated as $\widehat{f}(x)$ by fuzzy approach.

Adaptive-fuzzy control method has been divided to two parts: direct and indirect approachs; this paper uses indirect method.

2.1. Indirect Adaptive Fuzzy Controller (IAFC). First, consider the affine system equations given below:

$$
\begin{aligned}
& \dot{x}(t)=f(x)+g(x) u(t), \\
& \dot{x}(t)=(\alpha(t)+\alpha(x))+(\beta(t)+\beta(x)) * u(t) .
\end{aligned}
$$

$\alpha(x), \beta(x)$ are known parts of system dynamic (which are obtained from experimental and numerical methods), and $\alpha(t), \beta(t)$ are system equations that must be identified. Considering the above discussions, $\alpha(x)$ and $\beta(x)$ can be obtained as

$$
\begin{aligned}
& \alpha(x)=z_{\alpha}^{T} \cdot A_{\alpha}^{*} \cdot \xi_{\alpha}+d_{\alpha}(x), \\
& \beta(x)=z_{\beta}^{T} \cdot A_{\beta}^{*} \cdot \xi_{\beta}+d_{\beta}(x) .
\end{aligned}
$$

$d_{\alpha}(x), d_{\beta}(x)$ are approximated errors between real system and fuzzy system with upper bound values $\left|d_{\alpha}(x)\right| \leq D_{\alpha}(x)$, $\left|d_{\beta}(x)\right| \leq D_{\beta}(x)\left(D_{\alpha}(x), D_{\beta}(x)\right.$ are known values). So estimation of system parameters can be rewritten as follows:

$$
\begin{aligned}
& \widehat{\alpha}(x)=z_{\alpha}^{T} \cdot A_{\alpha} \cdot \xi_{\alpha}, \\
& \widehat{\beta}(x)=z_{\beta}^{T} \cdot A_{\beta} \cdot \xi_{\beta} .
\end{aligned}
$$


$A_{\alpha}, A_{\beta}$ are matrices that will be updated adaptively with the following updating laws:

$$
\begin{gathered}
\dot{A}_{\alpha}(t)=-Q^{-1} \cdot z \cdot \xi_{\alpha}^{T} \cdot \mathrm{es}, \\
\dot{A}_{\beta}(t)=-Q^{-1} \cdot z \cdot \xi_{\beta}^{T} \cdot \mathrm{es} \cdot u(t)
\end{gathered}
$$

and finally the control law is described as follows:

$$
u(t)=\frac{1}{\beta(t)+\widehat{\beta}(x)}(-[\alpha(t)+\widehat{\alpha}(x)]+v(t)) .
$$

2.2. Direct Adaptive Fuzzy Controller (DAFC). Control law $u(t)$ in feedback linearization method can be obtained as follows:

$$
u(t)=\frac{1}{\beta(x)}(-\alpha(x)+v(t)) .
$$

The goal is to estimate $u(t)$, with consideration of basic fuzzy function as seen below:

$$
\widehat{u}(t)=z_{u}^{T} \cdot A_{u}^{*} \cdot \xi_{u}+d_{u}(x),
$$

where $\widehat{u}(t)$ is the estimated signal and $d_{u}(x)$ is the error between the described $u$ by fuzzy method and desired (appropriate) $u^{*}$; this error is restricted by upper known bound $\left|d_{u}(x)\right| \leq D_{u}(x)$; since practically it is difficult to define $D_{u}(x)$, so its value is specified and adjusted in designing process repetition; this adjustment procedure continues until the performance of controller indicates that $D_{u}(x)$ comes close to proper value. Finally updating law $A_{u}(t)$ is obtained as follows:

$$
\dot{A}_{u}(t)=-Q_{u}^{-1} \cdot z_{u}^{T} \cdot \xi_{u} \cdot \text { es. }
$$

The initial value of $A_{u}(t)$ may be considered as $A_{u}(0)=0$.

Remark 1. This method is suitable for minimum phase plants and also has the following limitation for $\beta(x)$ :

$$
-\infty<\beta_{1}<\beta(x)<\beta_{2}<\infty
$$

\section{Proposed Approach}

In the previous section, an adaptive fuzzy controller using fuzzy basis functions is described for stabilizing the controlled system. In our introduced method, the same approach has been employed but our method does not need to utilize fuzzy basis functions for estimating system parameters. Instead, three proposed rules based on output errors are considered to control the plant.

Consider a system with general form

$$
\dot{x}(t)=f(x(t))+g(x(t)) u(t) .
$$

At first, nonlinear functions $f$ and $g$ must be estimated in such a way that controller does not rely on system dynamic. It should be noted that in this paper, the system is considered continues time but the output is sampled and these sampled points are used in proposed controller. The estimations of system dynamic can be defined as

$$
\begin{aligned}
& \tilde{f}(k)=\widehat{u}_{f}^{T}(k), \\
& \widetilde{g}(k)=\widehat{u}_{g}^{T}(k) .
\end{aligned}
$$

And the updating values of $\widehat{u}_{g}, \widehat{u}_{f}$ are given by

$$
\begin{gathered}
\widehat{u}_{f}(k+1)=\widehat{u}_{f}(k)+\alpha r^{T}(k+1), \\
\widehat{u}_{g}(k+1)= \begin{cases}\widehat{u}_{g}(k)+\beta u_{c}(k) r^{T}(k+1) & I=1 \\
\widehat{u}_{g}(k) & I=0,\end{cases}
\end{gathered}
$$

where $\alpha_{f}, \alpha_{g}$ are constant coefficients with respect to $\widehat{u}_{f}$, $\widehat{u}_{g}$. It should also be noted that usually these parameters do not need to be changed for different system dynamics. The value of $I$ will be defined in $(27) ; r(t)$ is the so-called "filtered tracking error" which is the sum of errors as defined below:

$$
\begin{aligned}
r(k)=e_{n}(k)+\lambda_{1} e_{n-1}(k)+\cdots+\lambda_{n-1} e_{2}(k)+\lambda_{n} e_{1}(k), \\
e_{1}(k)=y(k)-y_{d}(k), \\
e_{2}(k)=y(k)-y_{d}(k+1), \\
\vdots \\
e_{n}(k)=y(k)-y_{d}(k+n-1),
\end{aligned}
$$

where $\left\{\lambda_{i}\right\}, i=1, \ldots, n$ are coefficients of the predictive error. And $y_{d}$ is the desired output. For eliminating nonlinear terms of system dynamic, feedback linearization method has been exploited; for this purpose, $u(k)$ is chosen as follows:

$$
u(k)=\widetilde{g}^{-1}(k)(-\tilde{f}(k)+v(k)),
$$

where $v(k)$ is defined by

$$
v(k)=k_{v} r(k)+y_{d}(k+n)-\sum_{i=0}^{p-2} \lambda_{i+1} e_{n-i}(k) .
$$

In (23), $k_{v}$ is the tracking error coefficient. By choosing appropriate $k_{v}, r(k)$ is converged into zero and at last it causes $v(k)$ to be restricted. In other words, if the bigger $k_{v}$ is opted, better performance of output and quick tendency to the desired value are achieved while increasing the control signal $u$; it should be noted that if $k_{v}$ is chosen very large, it can make the system unstable.

As it is seen from (22), the problem of singularity occurs when $\tilde{g}$ is near zero and the control signal can become unbounded. To solve this problem, separate controller comprising two parts is proposed in the following form:

$u(k)$

$$
=\left\{\begin{array}{r}
u_{c}(k)+.5\left(u_{r}(k)-u_{c}(k)\right) \exp \left(\gamma\left(\left\|u_{c}(k)\right\|-s\right)\right) \\
I=1, \\
u_{r}(k)-.5\left(u_{r}(k)-u_{c}(k)\right) \exp \left(-\gamma\left(\left\|u_{c}(k)\right\|-s\right)\right) \\
I=0 .
\end{array}\right.
$$


In the above equation, $s$ is a constant value that its amount impacts on control signal $u . u_{c}, u_{r}$ are defined as

$$
\begin{aligned}
& u_{c}(k)=\widetilde{g}^{-1}(k)(-\tilde{f}(k)+v(k)), \\
& u_{r}(k)=-\mu\left\|u_{c}(k)\right\| \operatorname{sign} \frac{(r(k))}{\bar{g}},
\end{aligned}
$$

where $\mu$ is robust control signal coefficient that the decreasing or increasing of this coefficient affects quantity of final control signal $u$, and also $\bar{g}$ is a constant value that its small amount decreases the effect of $u_{r}$ on final control signal $u$; that is, $u_{r}$ will become more effective factor for determining $u$; and $I$ is given by the equation below:

$$
I= \begin{cases}1\|\widetilde{g}(k)\|>\bar{g}, & \left\|u_{c}(k)\right\|<s \\ 0, & \text { otherwise. }\end{cases}
$$

Here, $\bar{g}$ is lower bound of $\widetilde{g}$. Proper selection of $\bar{g}$ prevents $\widetilde{g}$ to be singular.

Remark 2. $\gamma, \mu$, and $s$ are designing parameters with the following conditions [5]: $\gamma<\ln (2 / s), \mu>0, s>0$.

Remark 3. If $u_{c}$ is greater than $1 / \gamma$, it is assumed to be equal to $1 / \gamma$.

The major principles of proposed MFA controller are constructed based on the three following experimental rules, which make controller produce appropriate control signal:

$$
u(k)= \begin{cases}u(k-1), & \text { if }\left|e_{1}(k)\right|<\varepsilon, \quad\left|e_{1}(k-1)\right|<\varepsilon, \\ & \left|e_{1}(k)\right| \leq\left|e_{1}(k-1)\right| \\ -u(k-1), & \text { if }\left|e_{1}(k)\right|<\varepsilon, \quad\left|e_{1}(k-1)\right|<\varepsilon \\ & \left|e_{1}(k)\right|>\left|e_{1}(k-1)\right| \\ -\eta u(k-1), & \text { if }\left|e_{1}(k)\right|>\left|e_{1}(k-1)\right|\end{cases}
$$

where $\varepsilon$ is threshold value for changing final control signal $u$ in the above rules. These laws utilize appropriate last level control signal (if error becomes less than specified value that is called $\varepsilon$ ) for using at next level. Indeed, if error at time $k$ and $k-1$ becomes less than $\varepsilon$ and also error at time $k$ is greater than the previous time, the controller applies negative value of previous control signal for current time.

Regarding adaptive fuzzy controllers $[5,7]$ that estimate unknown nonlinear functions of the system by fuzzy algorithm and when new reference input is applied to the system, this estimation becomes better. But the sensitivity of proposed controller on estimation of nonlinear functions is less than that of AFC method. This is because output error has significant role in control effort rather than significant role of estimation in AFC.

\section{Lyapunov Stability for the Proposed Model- Free Adaptive Controller}

Let the Lyapunov function candidate $V$ be given by [8]

$$
\begin{aligned}
V= & r^{T}(k) r(k)+\frac{1}{\alpha} \operatorname{tr}\left(\tilde{u}_{f}^{T}(k) \tilde{u}_{f}(k)\right) \\
& +\frac{1}{\beta} \operatorname{tr}\left(\tilde{u}_{g}^{T}(k) \tilde{u}_{g}(k)\right) .
\end{aligned}
$$

(a) For the first region, $I=1$.

At first we should obtain the difference of Lyapunov function in $k$ and $k+1$ level. So general Lyapunov function $\Delta V=\Delta V_{1}+\Delta V_{2}+\Delta V_{3}$ can be acquired as follows:

$$
\begin{gathered}
\Delta V_{1}=r^{T}(k+1) r(k+1)-r^{T}(k) r(k), \\
\Delta V_{2}=\frac{1}{\alpha} \operatorname{tr}\left(\tilde{u}_{f}^{T}(k+1) \tilde{u}_{f}(k+1)-\tilde{u}_{f}^{T}(k) \tilde{u}_{f}(k)\right), \\
\Delta V_{3}=\frac{1}{\beta} \operatorname{tr}\left(\tilde{u}_{g}^{T}(k+1) \tilde{u}_{g}(k+1)-\tilde{u}_{g}^{T}(k) \tilde{u}_{g}(k)\right) .
\end{gathered}
$$

If we prove that $\Delta V \leq 0$, it will demonstrate that $\tilde{u}_{g}, \tilde{u}_{f}$, and $r$ are bounded and they are able to stabilize system. $\Delta V_{1}, \Delta V_{2}$, and $\Delta V_{3}$ are obtained as follows:

$$
\begin{aligned}
\Delta V_{1}= & -r^{T}(k)\left(I-k_{v}^{T} k_{v}\right) r(k) \\
& +2\left(k_{v} r(k)\right)^{T}\left(\tilde{u}_{f}^{T}+\tilde{u}_{g}^{T} u_{c}+g u_{d}+\varepsilon^{\prime}\right) \\
& +\left(\tilde{u}_{f}^{T}\right)^{T} \tilde{u}_{f}^{T}+\left(\tilde{u}_{g}^{T} u_{c}\right)^{T}\left(\tilde{u}_{g}^{T} u_{c}\right) \\
& +\left(g u_{d}\right)^{T} g u_{d}+\varepsilon^{\prime T} \varepsilon^{\prime}+2 \tilde{u}_{f}\left(\tilde{u}_{g}^{T} u_{c}+g u_{d}+\varepsilon^{\prime}\right) \\
& +2\left(\tilde{u}_{g}^{T} u_{c}\right)^{T}\left(g u_{d}+\varepsilon^{\prime}\right)+2\left(g u_{d}\right)^{T} \varepsilon^{\prime}, \\
\Delta V_{2}= & -(2-\alpha)\left(\tilde{u}_{f}^{T}\right)^{T} \tilde{u}_{f}^{T} \\
& +\alpha\left(k_{v} r(k)+\tilde{u}_{g}^{T} u_{c}+g u_{d}+\varepsilon^{\prime}\right)^{T} \\
& \times\left(k_{v} r(k)+\tilde{u}_{g}^{T} u_{c}+g u_{d}+\varepsilon^{\prime}\right) \\
& -2(1-\alpha) \tilde{u}_{f}\left(k_{v} r(k)+\tilde{u}_{g}^{T} u_{c}+g u_{d}+\varepsilon^{\prime}\right), \\
\Delta V_{3}= & -(2-\beta)\left(\tilde{u}_{g}^{T} u_{c}\right)^{T}\left(\tilde{u}_{g}^{T} u_{c}\right) \\
& +\beta\left(k_{v} r(k)+\tilde{u}_{f}^{T}+g u_{d}+\varepsilon^{\prime}\right)^{T} \\
& \times\left(k_{v} r(k)+\tilde{u}_{f}^{T}+g u_{d}+\varepsilon^{\prime}\right) \\
& 2(1-\beta)\left(\tilde{u}_{g}^{T} u_{c}\right)^{T}\left(k_{v} r(k)+\tilde{u}_{f}^{T}+g u_{d}+\varepsilon^{\prime}\right) .
\end{aligned}
$$

With combination of the above three Lyapunov functions, (32) is established

$$
\begin{aligned}
\Delta V= & -r^{T}(k)\left(I-k_{v}^{T} k_{v}\right) r(k)-(1-\alpha)\left(\tilde{u}_{f}^{T}\right)^{T} \tilde{u}_{f}^{T} \\
& +2\left(k_{v} r(k)+g u_{d}+\varepsilon^{\prime}\right)^{T}\left(k_{v} r(k)+g u_{d}+\varepsilon^{\prime}\right)
\end{aligned}
$$




$$
\begin{aligned}
& +\alpha\left(k_{v} r(k)+\tilde{u}_{g}^{T} u_{c}+g u_{d}+\varepsilon^{\prime}\right)^{T} \\
& \times\left(k_{v} r(k)+\tilde{u}_{g}^{T} u_{c}+g u_{d}+\varepsilon^{\prime}\right) \\
& +2 \alpha \widetilde{u}_{f}\left(k_{v} r(k)+\tilde{u}_{g}^{T} u_{c}+g u_{d}+\varepsilon^{\prime}\right) \\
& -(1-\beta)\left(\tilde{u}_{g}^{T} u_{c}\right)^{T}\left(\tilde{u}_{g}^{T} u_{c}\right) \\
& +\beta\left(k_{v} r(k)+\tilde{u}_{f}^{T}+g u_{d}+\varepsilon^{\prime}\right)^{T} \\
& \times\left(k_{v} r(k)+\tilde{u}_{f}^{T}+g u_{d}+\varepsilon^{\prime}\right) \\
& +2 \beta\left(\tilde{u}_{g}^{T} u_{c}\right)^{T}\left(k_{v} r(k)+\tilde{u}_{f}^{T}+g u_{d}+\varepsilon^{\prime}\right) .
\end{aligned}
$$

By simplifying (32) and using the below definition,

$$
\eta= \begin{cases}\alpha+\beta\left\|u_{c}\right\|^{2} & I=1 \\ \alpha & I=0\end{cases}
$$

Inequality (34) is acquired as follows:

$$
\begin{aligned}
\Delta V \leq & -r^{T}(k)\left(I-(1+\eta) k_{v}^{T} k_{v}\right) r(k) \\
& +2 \eta\left(k_{v} r(k)\right)^{T}\left(g u_{d}+\varepsilon^{\prime}\right) \\
& +(1+\eta)\left(g u_{d}+\varepsilon^{\prime}\right)^{T}\left(g u_{d}+\varepsilon^{\prime}\right) \\
& -(1-\eta)\left\|\tilde{u}_{f}^{T}+\tilde{u}_{g}^{T} u_{c}-\frac{\eta}{1-\eta}\left(k_{v} r(k)+g u_{d}+\varepsilon^{\prime}\right)\right\|^{2} \\
& +\frac{\eta}{1-\eta}\left(k_{v} r(k)+g u_{d}+\varepsilon^{\prime}\right)^{T}\left(k_{v} r(k)+g u_{d}+\varepsilon^{\prime}\right)
\end{aligned}
$$

with assuming that $k_{v \text { max }}$ is the maximum value of $k_{v}$ and by resimplifying (34), inequality (35) is stated as given below:

$$
\begin{aligned}
\Delta V \leq & -\left(I-a_{1} k_{v \max }^{2}\right)\|r(k)\|^{2}+2 a_{2} k_{v \max }\|r(k)\|\left(g u_{d}+\varepsilon^{\prime}\right) \\
& +a_{1}\left(g u_{d}+\varepsilon^{\prime}\right)^{T}\left(g u_{d}+\varepsilon^{\prime}\right) \\
& -(1-\eta)\left\|\tilde{u}_{f}^{T}+\tilde{u}_{g}^{T} u_{c}-\frac{\eta}{1-\eta}\left(k_{v} r(k)+g u_{d}+\varepsilon^{\prime}\right)\right\|^{2},
\end{aligned}
$$

where $a_{1}$ and $a_{2}$ are described as

$$
a_{1}=1+\eta+\frac{\eta}{1-\eta}, \quad a_{2}=\eta+\frac{\eta}{1-\eta}
$$

By considering Lemma A.4 in Appendix A,

$$
\left\|g\left(u-u_{C}\right)+\varepsilon_{f}+\varepsilon_{g} u_{c}+d\right\|=\left\|g u_{d}+\varepsilon^{\prime}\right\| \leq c_{0}+c_{1}\|r(k)\| .
$$

And with substituting inequality (37) in (35),

$$
\begin{aligned}
\Delta V \leq & -\left(I-a_{1} k_{v \max }^{2}\right)\|r(k)\|^{2} \\
& +2 a_{2} k_{v \max }\|r(k)\|\left(c_{0}+c_{1}\|r(k)\|\right) \\
& +a_{1}\left(c_{0}+c_{1}\|r(k)\|\right)^{T}\left(c_{0}+c_{1}\|r(k)\|\right) \\
& -(1-\eta)\left\|\tilde{u}_{f}^{T}+\tilde{u}_{g}^{T} u_{c}-\frac{\eta}{1-\eta}\left(k_{v} r(k)+g u_{d}+\varepsilon^{\prime}\right)\right\|^{2} .
\end{aligned}
$$

With simplifying the above inequality, the below equation is obtained:

$$
\begin{aligned}
\Delta V \leq & -\left(I-a_{3}\right)\|r(k)\|^{2}+2 a_{4}\|r(k)\|+a_{5} \\
& -(1-\eta)\left\|\tilde{u}_{f}^{T}+\tilde{u}_{g}^{T} u_{c}-\frac{\eta}{1-\eta}\left(k_{v} r(k)+g u_{d}+\varepsilon^{\prime}\right)\right\|^{2},
\end{aligned}
$$

where $a_{3}, a_{4}$, and $a_{5}$ are defined as

$$
\begin{aligned}
& a_{3}=a_{1} k_{v \max }^{2}+2 a_{2} c_{1} k_{v \max }+a_{1} c_{1}^{2}, \\
& a_{4}=a_{2} c_{0} k_{v \max }+a_{1} c_{0} c_{1}, \\
& a_{5}=a_{1} c_{0}^{2} .
\end{aligned}
$$

By assuming that $\eta<1$, the below statement becomes negative:

$$
-(1-\eta)\left\|\tilde{u}_{f}^{T}+\widetilde{u}_{g}^{T} u_{c}-\frac{\eta}{1-\eta}\left(k_{v} r(k)+g u_{d}+\varepsilon^{\prime}\right)\right\|^{2}<0 .
$$

As regards $\alpha>0, \beta>0, \eta$ becomes positive too and finally $a_{1}, a_{2}, a_{3}, a_{4}, a_{5}>0$ totally; the first term in (39) $\{-(I-$ $\left.\left.a_{3}\right)\|r(k)\|^{2}+2 a_{4}\|r(k)\|+a_{5}\right\}$ becomes nonpositive, if it obeys the below condition:

$$
\|r(k)\|>\delta_{r 1}
$$

in which $\delta_{r 1}$ is acquired from the below inequality:

$$
\left\|\delta_{r 1}\right\|>\frac{a_{5}+\sqrt{a_{5}^{2}+a_{6}\left(1-a_{4}\right)}}{\left(1-a_{4}\right)} .
$$

Thus, both terms of inequality (39) have nonpositive values and finally $\Delta V \leq 0$.

(b) For the second region, $I=0$. 
In this region the proposed Lyapunov functions $\Delta V_{1}$, $\Delta V_{2}$, and $\Delta V_{3}$ are obtained like (30) and they are stated as follows:

$$
\begin{aligned}
\Delta V_{1}= & -r^{T}(k)\left(I-k_{v}^{T} k_{v}\right) r(k) \\
& +2\left(k_{v} r(k)\right)^{T}\left(\tilde{u}_{f}^{T}+\overline{g u}_{d}+\varepsilon^{\prime}\right)+\left(\widetilde{u}_{f}^{T}\right)^{T} \widetilde{u}_{f}^{T} \\
& +\left(\overline{g u}_{d}\right)^{T} \overline{g u}_{d}+\varepsilon^{\prime T} \varepsilon^{\prime}+2 \widetilde{u}_{f}\left(\overline{g u}_{d}+\varepsilon^{\prime}\right)+2\left(\overline{g u}_{d}\right)^{T} \varepsilon^{\prime}, \\
\Delta V_{2}=- & (2-\alpha)\left(\widetilde{u}_{f}^{T}\right)^{T} \widetilde{u}_{f}^{T} \\
& +\alpha\left(k_{v} r(k)+\bar{g}_{d}+\varepsilon^{\prime}\right)^{T}\left(k_{v} r(k)+\overline{g u}_{d}+\varepsilon^{\prime}\right) \\
& -2(1-\alpha) \tilde{u}_{f}\left(k_{v} r(k)+\overline{g u}_{d}+\varepsilon^{\prime}\right),
\end{aligned}
$$

$\Delta V_{3}=0$

By combination of the above three equations (and definition of $\eta$ ), the statement is demonstrated below:

$$
\begin{aligned}
\Delta V= & -r^{T}(k)\left(I-k_{v}^{T} k_{v}\right) r(k) \\
& +2\left(k_{v} r(k)+\overline{g u}_{d}+\varepsilon^{\prime}\right)^{T}\left(\bar{g}_{d}+\varepsilon^{\prime}\right) \\
& +\frac{1}{1-\alpha}\left(k_{v} r(k)+\overline{g u}_{d}+\varepsilon^{\prime}\right)^{T}\left(k_{v} r(k)+\overline{g u}_{d}+\varepsilon^{\prime}\right) \\
& -(1-\eta)\left\|\tilde{u}_{f}^{T}-\frac{\eta}{1-\eta}\left(k_{v} r(k)+\overline{g u}_{d}+\varepsilon^{\prime}\right)\right\|^{2}
\end{aligned}
$$

with the consideration of Lemma A.4

$$
\left\|g u-\widehat{g} u_{C}\right\| \leq d_{0}+d_{1}\|r(k)\| .
$$

By substituting inequality (46) in (45),

$$
\begin{aligned}
\Delta V \leq & -\left(I-b_{0}\right)\|r(k)\|^{2}+2 b_{1}\|r(k)\|+b_{2} \\
& -(1-\eta)\left\|\tilde{u}_{f}^{T}-\frac{\eta}{1-\eta}\left(k_{v} r(k)+\overline{g u}_{d}+\varepsilon^{\prime}\right)\right\|^{2}
\end{aligned}
$$

in which $b_{0}, b_{1}$, and $b_{2}$ are as follows:

$$
\begin{aligned}
& b_{0}=k_{v \max }^{2}+2 d_{1}\left(d_{1}+k_{v \max }\right)+\frac{\left(d_{1}+k_{v \max }\right)^{2}}{1-\alpha}, \\
& b_{1}=2\left(d_{0}+\varepsilon^{\prime}\right) d_{1}+\frac{4-2 \alpha}{1-\alpha}\left(d_{0}+\varepsilon^{\prime}\right)\left(d_{1}+k_{v \max }\right), \\
& b_{2}=\frac{3-2 \alpha}{1-\alpha}\left(d_{0}+\varepsilon^{\prime}\right)^{2} .
\end{aligned}
$$

By the assumption that $\eta<1$, the statement below becomes negative:

$$
-(1-\eta)\left\|\widetilde{u}_{f}^{T}-\frac{\eta}{1-\eta}\left(k_{v} r(k)+\overline{g u}_{d}+\varepsilon^{\prime}\right)\right\|^{2}<0 .
$$

As regards $\alpha>0, \beta>0, \eta$ becomes positive too and finally $b_{0}$, $b_{1}, b_{2}>0$. The first term in (39) $\left\{-\left(I-b_{0}\right)\|r(k)\|^{2}+2 b_{1}\|r(k)\|+\right.$ $b_{2}$ \} becomes nonpositive, if it obeys the below condition:

$$
\|r(k)\|>\delta_{r 2}
$$

in which $\delta_{r 2}$ is obtained from the relation below:

$$
\left\|\delta_{r 2}\right\|>\frac{b_{1}+\sqrt{b_{1}^{2}+b_{2}\left(1-b_{0}\right)}}{\left(1-b_{0}\right)} .
$$

As a result, both terms of inequality (47) have nonpositive values which means $\Delta V \leq 0$. And finally if $\|r(k)\|>$ $\max \left(\delta_{r 1}, \delta_{r 2}\right)$ Lyapunov function becomes negative.

\section{Implementation of MFA Controller}

Example 4. Consider MIMO system with 2 input-2 output system state equations which are given below [8]:

$$
\begin{gathered}
\dot{x}_{1}(t)=x_{2}(t)+\left(1+x_{2}(t)^{2}\right) u_{1}(t)+d_{1}(t), \\
\dot{x}_{2}(t)=-x_{1}(t)-\left(0.1-\exp \left(-x_{1}(t)^{2}-x_{2}(t)^{2}\right)\right) x_{2}(t) \\
+\left(1+x_{1}(t)^{2}\right) u_{2}(t)+d_{2}(t), \\
y_{1}(t)=x_{1}(t)+n_{1}(t), \quad y_{2}(t)=x_{2}(t)+n_{2}(t), \\
n(t)=\text { measurement noise, } \\
d_{1}(t), d_{2}(t)=\text { disturbance. }
\end{gathered}
$$

Select control parameters as follows:

$$
\begin{gathered}
\mu=10, \quad \alpha=.1, \quad \beta=.1, \quad s=2, \quad \gamma=.05, \\
\bar{g}=.8, \quad k_{v}=\left[\begin{array}{lll}
10 & 0 ; 0 & 10
\end{array}\right], \quad \lambda_{1}=.001, \quad \varepsilon=.01, \\
\eta=0, \quad \widehat{u}_{f}=\left[\begin{array}{ll}
.1 & .1
\end{array}\right], \quad \widehat{u}_{g}=\left[\begin{array}{lll}
1 & 0 ; & 0
\end{array}\right] .
\end{gathered}
$$

Simulation results of MIMO system without disturbances $d_{1}(t), d_{2}(t)=0$ are demonstrated in Figure 1. In Figure 2 external disturbances are applied to the system with power $d_{1}(t)=0.4, d_{2}(t)=0.4$, and finally, the system with existence of white Gaussian measurement noise (power 0.0001) and external disturbance is shown in Figure 3; also desired inputs are assumed: $y_{1 d}=1, y_{2 d}=\cos (2 \pi t)$.

The aim of using this MIMO system which is brought by [8] (along with two control signals) is surveying coupling effect in multiinput multioutput systems. As it is understood from the result of Figure 1 caused by MFA controller and the result of AFC in [8], it can be said that system outputs of MFA controller do not have any overshoot in its response but in AFC, this problem is observed. As it is seen from Figure 2, MFA controller rejects disturbance without any noticeable effort in control signals and in the outputs. Also it can be deduced from Figure 3 that the measurement noise has intense effect on system outputs and control signals 


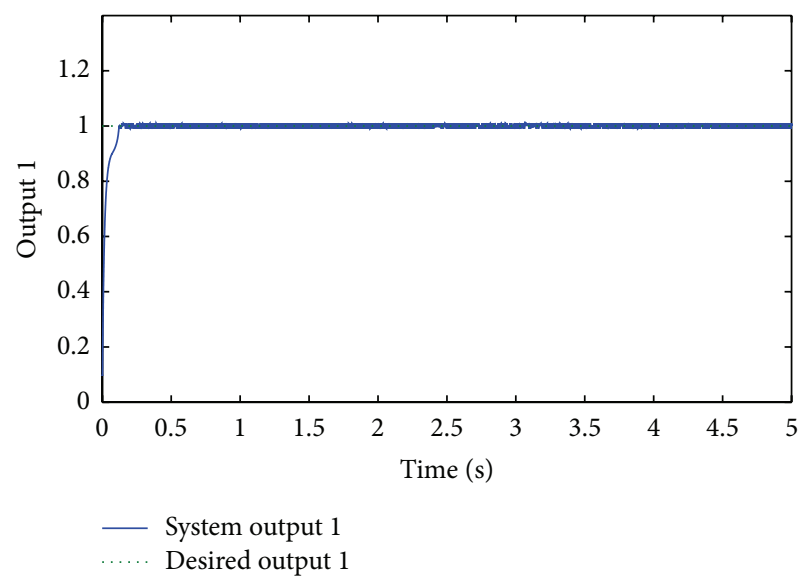

(a)

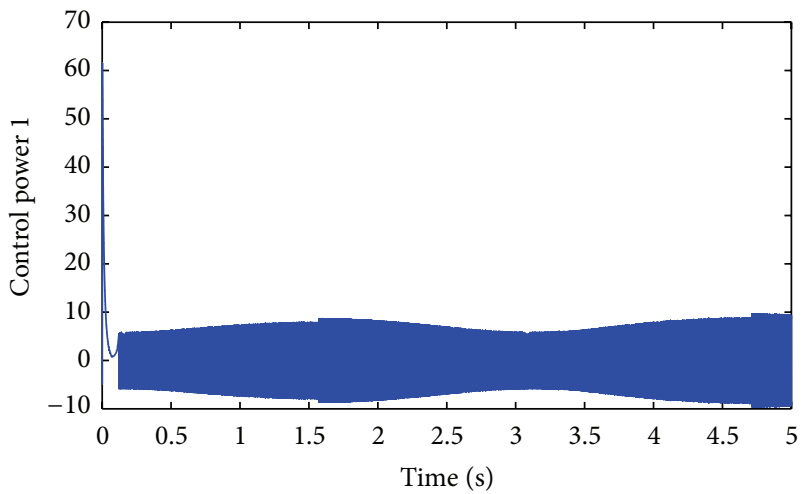

(c)

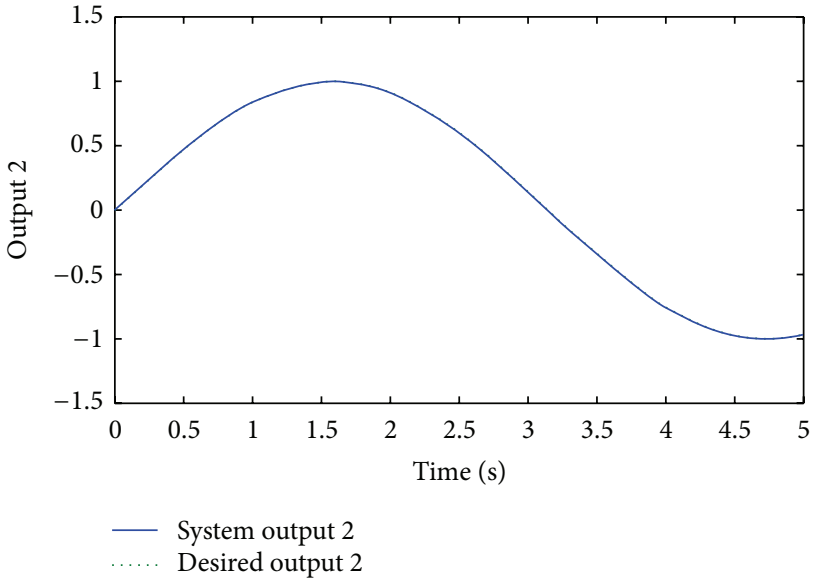

(b)

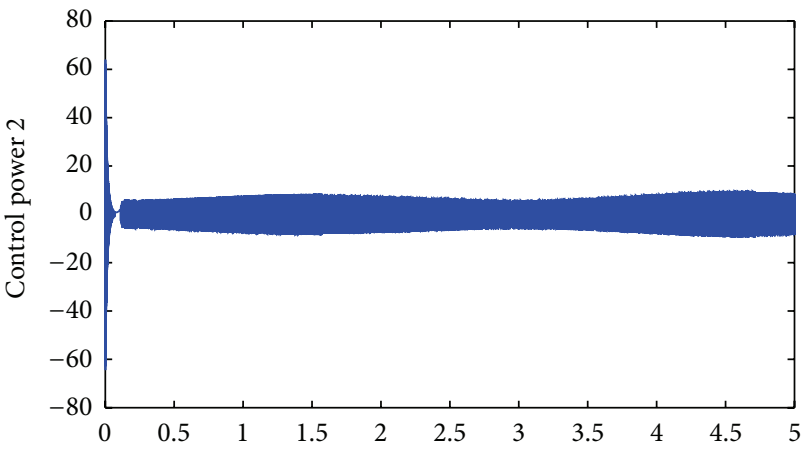

(d)

FIGURE 1: Simulation results for MIMO system without disturbance. (a), (b) system outputs 1 and 2. (c), (d) control signals $u$ relative to inputs 1 and 2 .

(approximately became twice) but it should be noted that the proposed controller is able to track desired output and yet remains stable. As it is demonstrated in Figures 1, 2, and 3, the disadvantage of this controller is oscillating control signal. Profits of MFAC in addition of good (also rapid) tracking and system stabilizing are decreasing settling time of output response.

Example 5. The second system under study is Maglev train which is shown in Figure 4. Using the notations given in Figure 4 , the vertical dynamics is described by [9].

System state equations are described as follows:

$$
\begin{aligned}
m \frac{d^{2} z(t)}{d t^{2}} & =-F(i, z, t)+f_{d}+m g \\
& =-\frac{\mu_{0} N^{2} a_{m}}{4}\left[\frac{i(t)}{z(t)}\right]^{2}+f_{d}+m g, \\
\frac{d i(t)}{d t}= & \frac{i(t)}{z(t)} \frac{d z(t)}{d t} \\
& -\frac{2}{\mu_{0} N^{2} a_{m}} z(t)\left(R_{m} i(t)-u(t)\right) .
\end{aligned}
$$

Here $z$ is the distance between rail and train, $i$ is the current of windings, $m$ is the train mass, $f_{d}$ is the force of disturbance, $\mu_{0}$ is the permeability coefficient which equals $4 \pi * 10^{-7}, N$ the number of turns in winding, $a_{m}$ is the section surface of windings, and $R_{m}$ is the wire resistance in windings.

Consider the state vector as below; thus the above equations convert to new state equations:

$$
\begin{aligned}
\dot{x}_{1}(t)= & x_{2}(t), \\
\dot{x}_{2}(t)= & -\frac{\mu_{0} N^{2} a_{m}}{4 m}\left[\frac{x_{3}(t)}{x_{1}(t)}\right]^{2}+\frac{1}{m} w+g, \\
\dot{x}_{3}(t)= & -\frac{2 R_{m}}{\mu_{0} N^{2} a_{m}} x_{3}(t) x_{1}(t)+\frac{x_{2}(t) x_{3}(t)}{x_{1}(t)} \\
& \quad+\frac{2 x_{1}(t) u(t)}{\mu_{0} N^{2} a_{m}} \\
y(t)= & x_{2}(t)+n(t) \\
n(t)= & \text { measurement noise, } \\
w(t)= & \text { external disturbance. }
\end{aligned}
$$




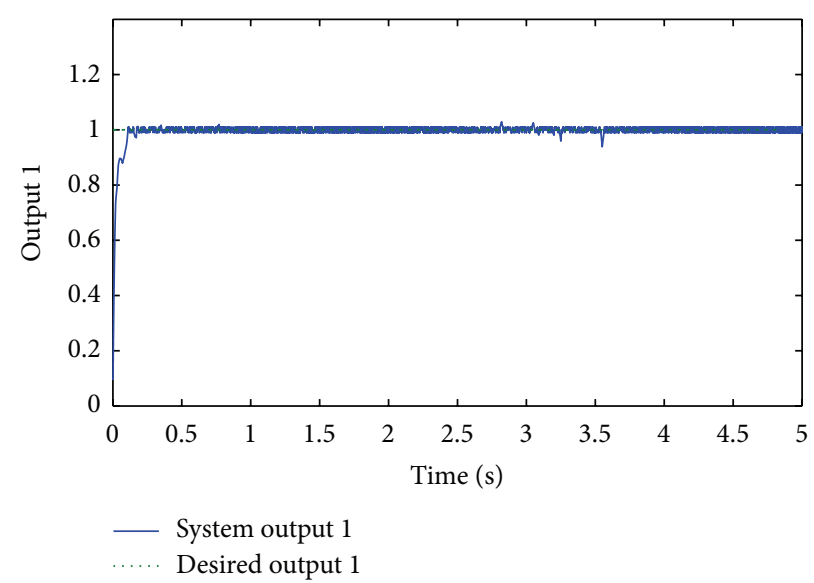

(a)

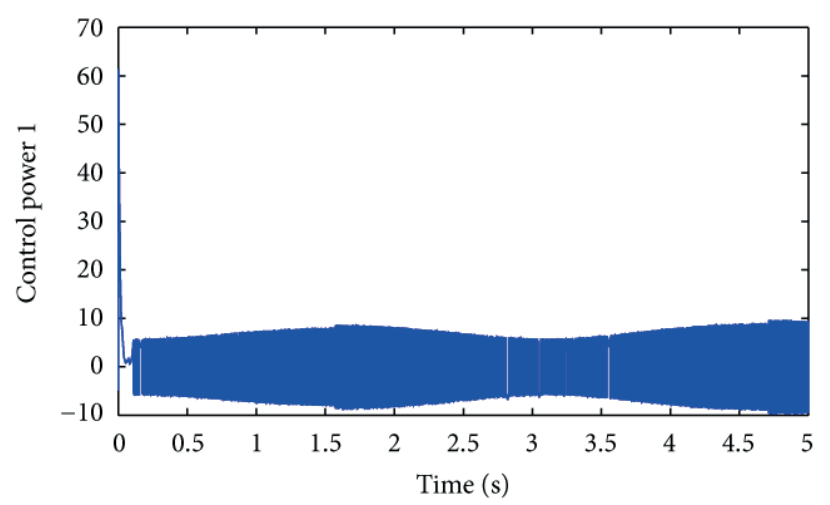

(c)

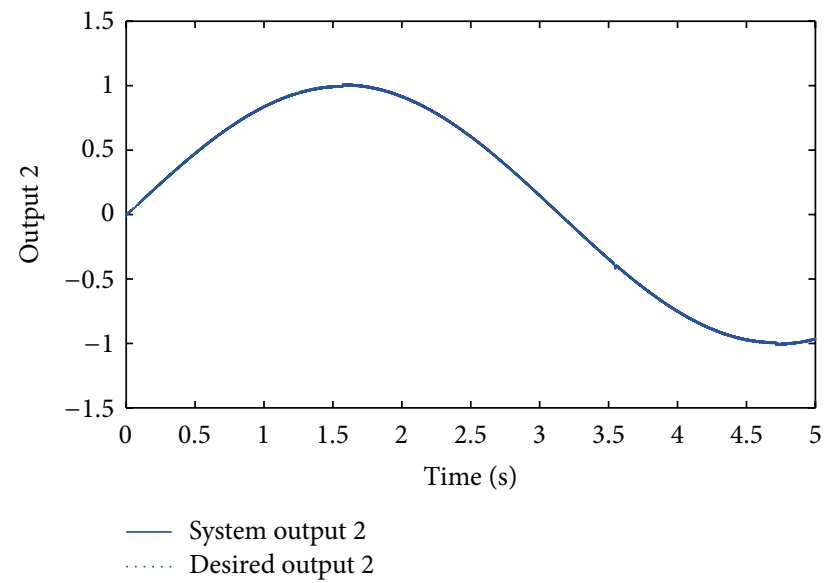

(b)

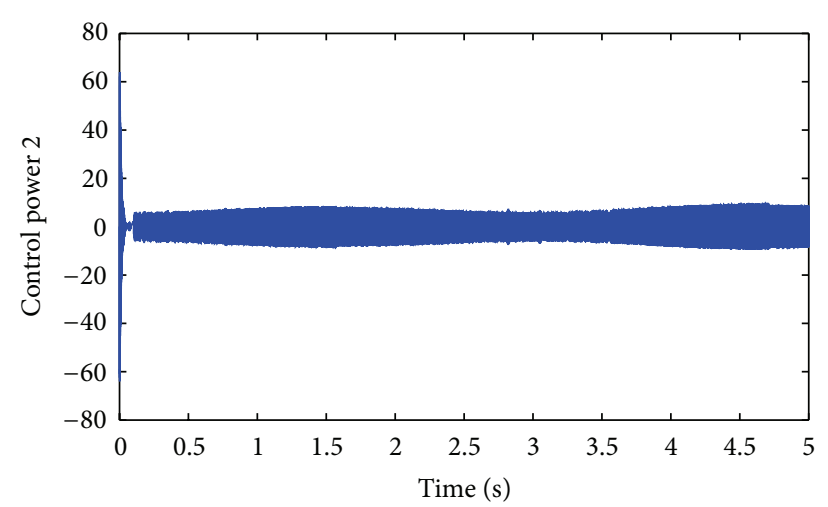

(d)

FIGURE 2: Simulation results for MIMO system with disturbance. (a), (b) system outputs 1 and 2. (c), (d) control signals $u$ relative to inputs 1 and 2 .

Maglev trains work in one of two ways; both methods are based on the same concept but involve different approaches.

(1) Electromagnetic Suspension is based on magnetic attraction; it is very complex and somewhat unstable.

(2) Electrodynamic Suspension is based on the repulsion of magnets. The magnetic levitation force balances the weight of the car at a stable position. Controlling EMS is more difficult than EDS train, because normality of this dynamic state is unstable.

Magnetic trains have two important issues, levitation and propulsion; the target of controller is first: goes up train to desired level along with guarantee stabilizing against some uncertainty such as wind and changing train mass in boltroads. And second adjust train speed at the working frequency of magnets. In this paper just levitation part (important section of train in controlling) is discussed. The train in this example goes from $10 \mathrm{~mm}$ to $16 \mathrm{~mm}$ level. By considering these assumption values for train and controller as follows,

$$
\begin{gathered}
m=1.5 \mathrm{~kg}, \quad N=280 \text { turns, } \quad a_{m}=102.4 \mathrm{~m}^{2}, \\
R_{m}=1.1 \Omega, \quad \mu=32, \quad \alpha=.1, \quad \beta=.1, \\
s=140, \quad \gamma=.01, \quad \bar{g}=.9, \quad k_{v}=1, \quad \lambda_{1}=0, \\
\varepsilon=.0001, \quad \eta=1, \quad \widehat{u}_{f}=-4, \quad \widehat{u}_{g}=1 .
\end{gathered}
$$

The result of simulating without considering disturbance has been displayed in Figure 5; in Figure 6, input step as external disturbance with amplitude $d(t)=1$ is applied to the system; for Figure 7, sinusoidal measurement noise with amplitude $.1 \mathrm{~mm}$ in presence of disturbance is considered. Also white Gaussian noise in existence of external disturbance is applied to the system with power $8 \times 10^{-9}$ (it is considered small because $I / O$ amplitude is small) which is shown in Figure 8. The purpose of presenting this system is studying on sort of complicated practical system dynamics such as Maglev in which they have some 


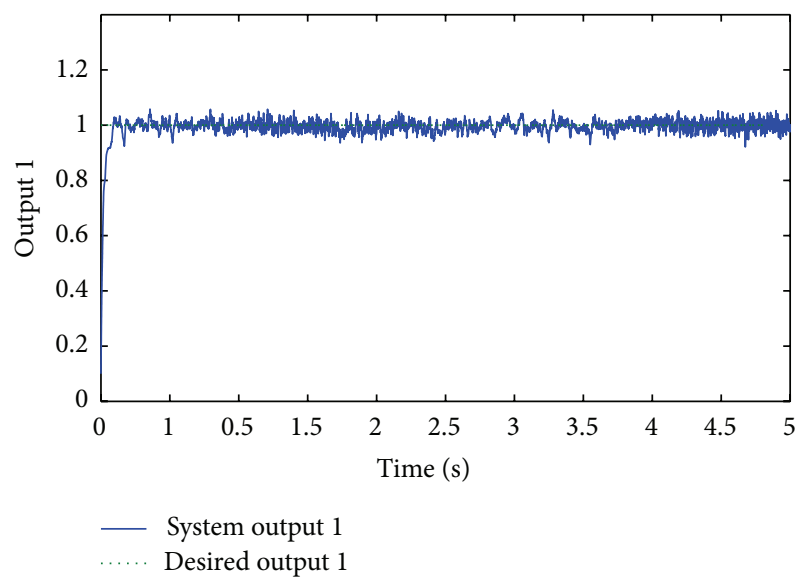

(a)

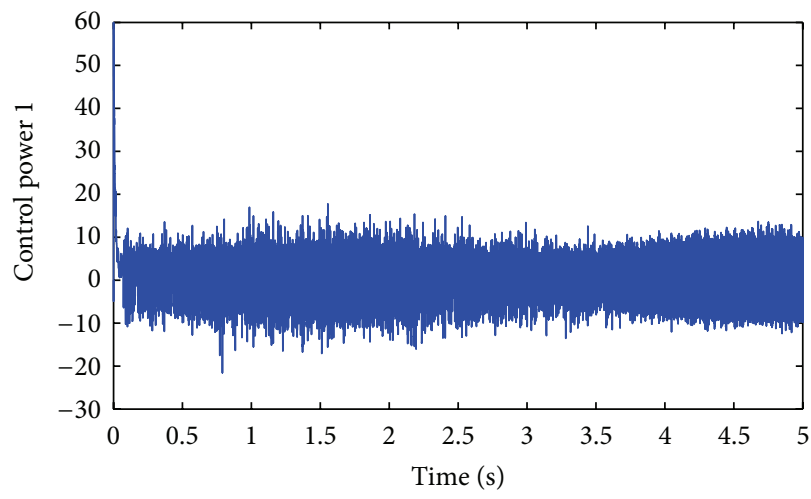

(c)

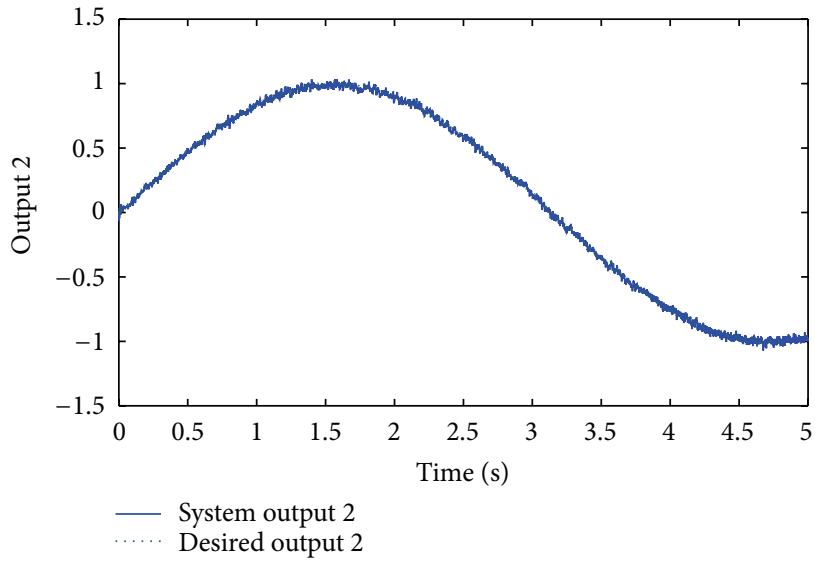

(b)

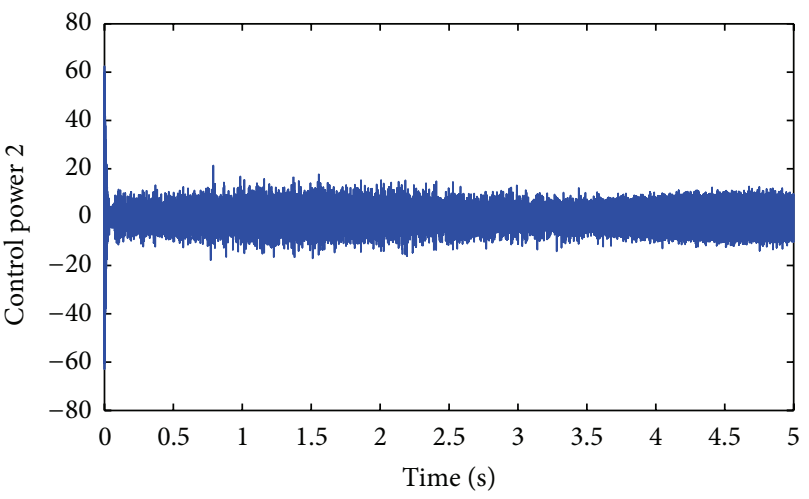

(d)

FIGURE 3: Simulation results for MIMO system with the measurement noise and disturbance. (a), (b) system outputs 1 and 2. (c), (d) control signals $u$ relative to inputs 1 and 2 .

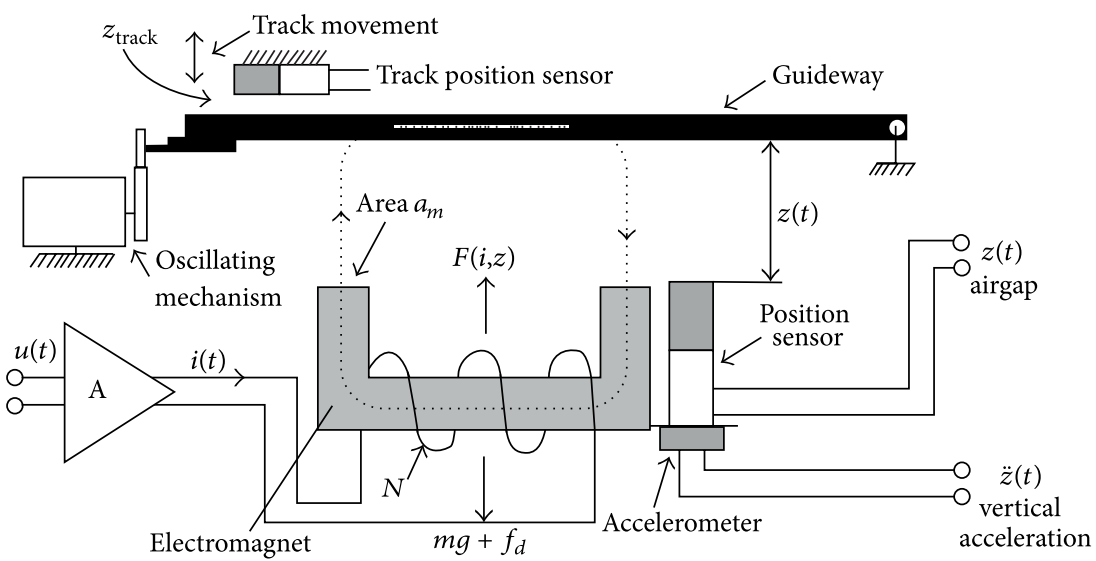

FIGURE 4: Vertical slice of magnetic levitation and rail in a magnetic train [9].

nonlinear terms like existence of first state in denominator (that affect directly the destabilizing system), and so forth. As it is demonstrated in Figure 5(a), the system output, that is distance between rail and train, has no overshoot; after about .25 second it goes from 10 to $16 \mathrm{~mm}$ smoothly. Such as the last example, it is acquired from Figure 6 that disturbance did not have any effect on the output, control effort, and settling time. In Figure 7, it is illustrated that sinusoidal measurement noise with no changes appears into output but no noticeable alternation in control signal has been seen. And finally in Figure 8, an impalpable effect of white Gaussian measurement noise on output and control signal can be shown; in this example some repeated benefits which are seen in Maglev system response are 


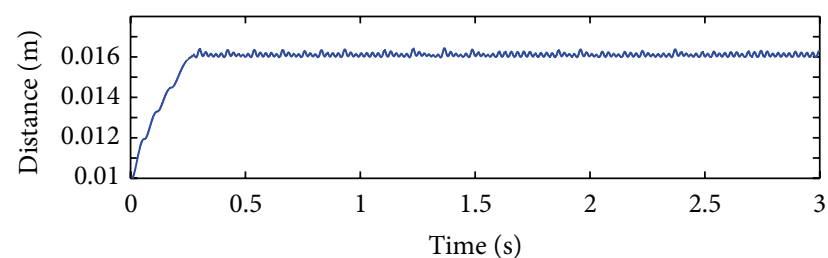

(a)

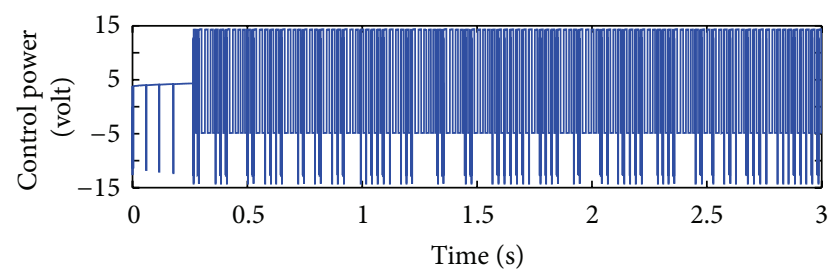

(b)

FIGURE 5: Simulation result for Maglev without disturbance (in which it goes from 10 to $16 \mathrm{~mm}$ ). (a) Distance between train and rail, (b) control signal.

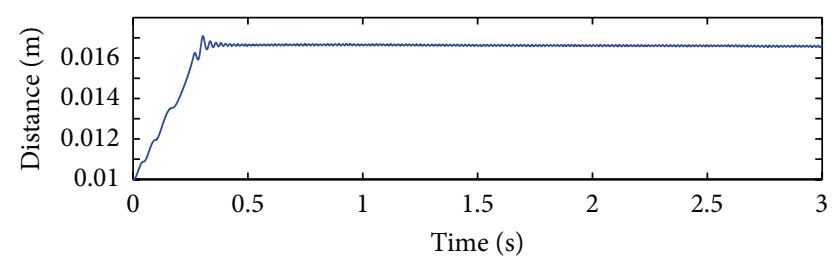

(a)

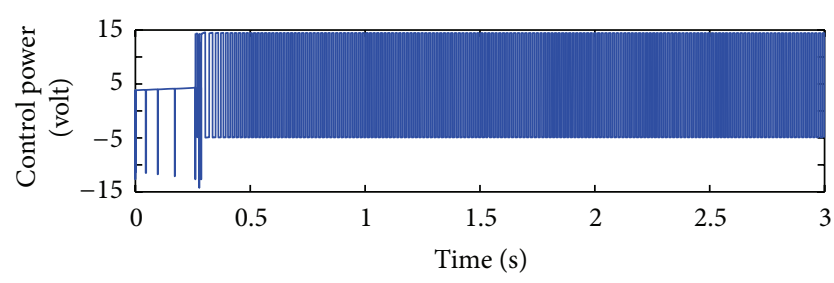

(b)

Figure 6: Simulation result for Maglev with disturbance. (a) Distance between train and rail, (b) control signal.

expressed, such as appropriate system stabilizing, good and rapid tracking, disturbance rejection, and small settling time.

\section{Conclusion}

Model-free adaptive controller is an adaptive controller that just uses system outputs and does not require another system states (so does not need observer) and with this, as modelfree controller, it performs good and also has some merits such as good stability, appropriate tracking, robust against uncertainty, disturbance rejection, and good decoupling and the biggest advantage of MFA controller is no requiring to system dynamic and even does not need to any prior experiment about system.

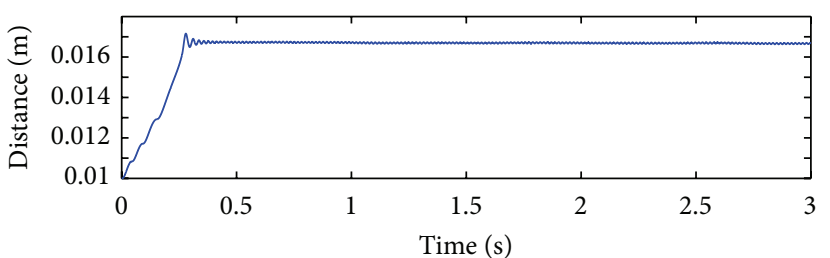

(a)

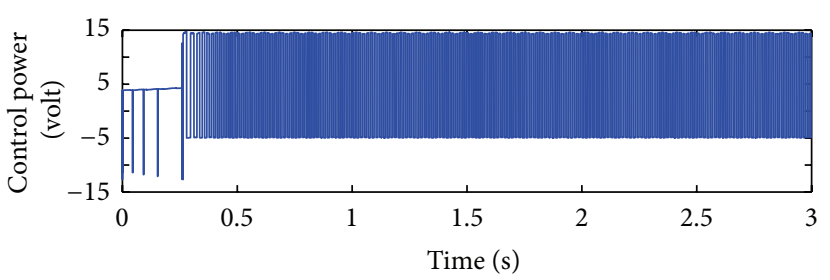

(b)

FIGURE 7: Simulation result for Maglev with sinuous measurement noise at $15 \mathrm{~Hz}$ and disturbance. (a) Distance between train and rail, (b) control signal.

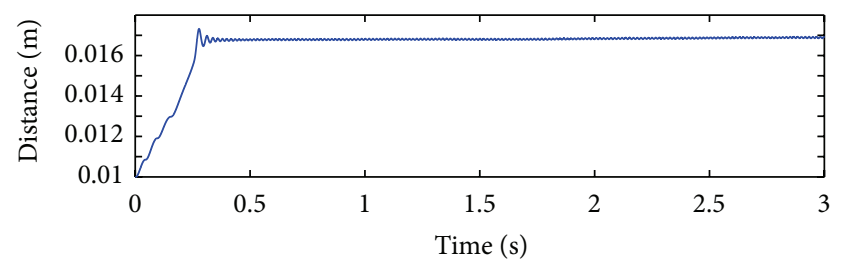

(a)

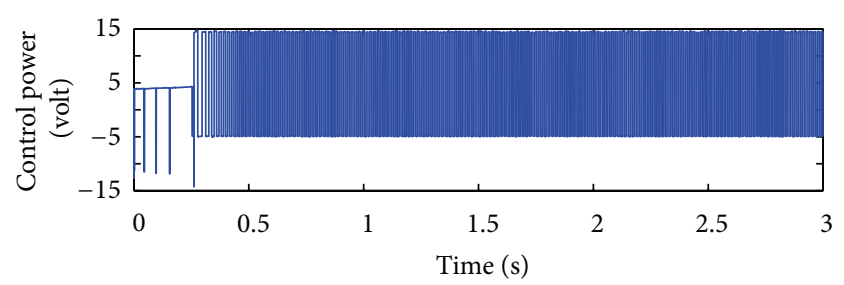

(b)

FIGURE 8: Simulation result for Maglev train with white Gaussian measurement noise along with disturbance. (a) Distance between train and rail, (b) control signal.

\section{Appendices}

\section{A. Some Requirements}

Lemma A.1. For each time $k, x(k)$ is bounded by

$$
\|x(k)\| \leq d_{0}+d_{1}\|r(k)\|
$$

which is $r(k)$, called "tracking error," and $d_{0}, d_{1}$ are computable positive constants. 
Proof [see [8, Lemma 3.2]]. Let $x(k) \in U$, in which $U$ is a compact subset of $R^{n}$. Assume that $h(x(k)) \in C^{\infty} U$; that is, $h(x(k))$ is a smooth function $U \rightarrow R$, so that the Taylor series expansion of $h(x(k))$ exists. Then using the bound on $x(k)$, express the function $h(x(k))$ on a compact set as linear form of parameters. Thus, the upper bound for $h(x(k))$ can be obtained as

$$
\|h(x(k))\| \leq c_{0}+c_{1}\|r(k)\|,
$$

where $c_{0}$ and $c_{1}$ are constant matrices [8].

Remark A.2. $\varepsilon_{g}, \varepsilon_{f}$ are estimation errors of $g, f$, respectively, and $d$ is external disturbance. These errors are bounded and have these definite bounds

$$
\left\|\varepsilon_{f}\right\| \leq \varepsilon_{N f}, \quad\left\|\varepsilon_{g}\right\| \leq \varepsilon_{N g}, \quad\|d\| \leq d_{M} .
$$

Remark A.3. According to (27), if $I=1$ then $\left\|u_{c}\right\| \leq s$, so $u_{c}$ is bounded and if $I=0$ then $\left\|u_{c}\right\|>s$, but by considering the mentioned condition at Remark 3 in Section 5, in which if $u_{c}$ is greater than $1 / \gamma$, it is assumed to be equal $1 / \gamma$. Thus, it can be deduced that in both regions and in all of times, $u_{c}$ is bounded and its bounds are shown as follows:

$$
\left\|u_{c}\right\| \leq \begin{cases}s & I=1, \\ \frac{1}{\gamma} & I=0 .\end{cases}
$$

Lemma A.4. With regards to that, the proposed control algorithm is divided into two regions $I=0$ and $I=1$. We will prove the following relationship which is established in each region:

$$
\begin{cases}\left\|g\left(u-u_{C}\right)+\varepsilon_{f}+\varepsilon_{g} u_{c}+d\right\| \leq c_{0}+c_{1}\|r(k)\| & I=1, \\ \left\|g u-\hat{g} u_{C}\right\| \leq d_{0}+d_{1}\|r(k)\| & I=0 .\end{cases}
$$

Proof. (a) First region: $I=1$ or $\left\|u_{c}\right\| \leq s$ and $\|g(x)\|>\bar{g}$.

In consideration of Lemma A.1, it is demonstrated that $\|g(x)\| \leq c_{01}+c_{11}\|r(k)\|$, where $c_{01}, c_{11}$ are constant matrices:

$$
\left\|u_{c}\right\| \leq s \longrightarrow\left\|u_{r}\right\| \leq \frac{\mu}{\bar{g}}\left\|u_{c}\right\| \longrightarrow \text { bounded }\left\|u_{r}\right\| .
$$

By using the result of relation (A.6), the following inequality is obtained:

$$
\left\|u-u_{c}\right\| \leq\left\|\frac{u_{r}-u_{c}}{2} e^{\gamma\left(\left\|u_{c}\right\|-s\right)}\right\| .
$$

As regards $\left\|u_{c}\right\| \leq s \rightarrow \gamma\left(\left\|u_{c}\right\|-s\right) \leq 0$,

$$
\left\|u-u_{c}\right\| \leq\left\|\frac{u_{r}-u_{c}}{2}\right\| \leq \frac{1}{2}\left(\left\|u_{r}\right\|+\left\|u_{c}\right\|\right) \leq c_{2} .
$$

By considering (A.7),

$$
\begin{aligned}
\left\|g\left(u-u_{C}\right)\right\| & \leq\|g\|\left\|\left(u-u_{C}\right)\right\| \\
& \leq c_{2}\left(c_{01}+c_{11}\|r(k)\|\right)=c_{02}+c_{12}\|r(k)\| .
\end{aligned}
$$

Also by utilizing Remark 3, (A.10) is stated as follows:

$$
\begin{aligned}
\left\|\varepsilon_{f}+\varepsilon_{g} u_{c}+d\right\| & \leq\left\|\varepsilon_{f}\right\|+\left\|\varepsilon_{g}\right\|\left\|u_{c}\right\|+\|d\| \\
& \leq \varepsilon_{N f}+\varepsilon_{N g}+d_{M}=\varepsilon_{N} .
\end{aligned}
$$

By using (A.9) and (A.10) the following inequality is acquired:

$$
\begin{aligned}
& \left\|g\left(u-u_{C}\right)+\varepsilon_{f}+\varepsilon_{g} u_{c}+d\right\| \\
& \quad \leq\left\|g\left(u-u_{C}\right)\right\|+\left\|\varepsilon_{f}+\varepsilon_{g} u_{c}+d\right\| \\
& \quad \leq c_{02}+c_{12}\|r(k)\|+\varepsilon_{N} \leq c_{0}+c_{1}\|r(k)\| .
\end{aligned}
$$

Thus, the assumption is proved in the first region.

(b) Second region: $I=0$ or $\left\|u_{c}\right\| \geq s$ and $\|g(x)\|<\bar{g}$ and the mentioned condition for the proposed MFA controller in Remark 3 in Section 5 is stated as follows:

$$
\text { if } u_{c}>\frac{1}{\gamma} \Longrightarrow u_{c}=\frac{1}{\gamma} \text {. }
$$

In consideration of Lemma A.1, it is demonstrated that $\|g(x)\| \leq c_{01}+c_{11}\|r(k)\|$, where $c_{01}, c_{11}$ are constant matrices. By using the result of relation (A.6) and the condition that is given above, the following inequality is obtained:

$$
\|u\| \leq\left\|u_{r}\right\|+\left\|\frac{u_{r}-u_{c}}{2} e^{-\gamma\left(\left\|u_{c}\right\|-s\right)}\right\| .
$$

As regards $\left\|u_{c}\right\|>s \rightarrow-\gamma\left(\left\|u_{c}\right\|-s\right) \leq 0$,

$$
\|u\| \leq\left\|u_{r}\right\|+\left\|\frac{u_{r}-u_{c}}{2}\right\| \leq \frac{1}{2}\left(3\left\|u_{r}\right\|+\left\|u_{c}\right\|\right) \leq d_{2} .
$$

By considering (B.5),

$$
\begin{aligned}
\|g u\| & \leq\|g\|\|u\| \leq d_{2}\left(c_{01}+c_{11}\|r(k)\|\right) \\
& =d_{02}+d_{12}\|r(k)\|, \\
\left\|\hat{g} u_{c}\right\| & \leq\|\bar{g}\|\left\|\frac{1}{\gamma}\right\| \leq d_{3} .
\end{aligned}
$$

By using (A.10) and (A.15), the following inequality is obtained:

$$
\begin{aligned}
\left\|g u-\widehat{g} u_{c}\right\| & \leq\|g u\|+\left\|\hat{g} u_{c}\right\| \\
& \leq d_{01}+d_{11}\|r(k)\|+d_{3} \\
& \leq d_{0}+d_{1}\|r(k)\| .
\end{aligned}
$$

Finally, the assumption is proved in the second region. Thus, inequality (A.5) is correct in both regions.

\section{B. Obtain $r(k)$}

In consideration of error definition $r(k)$ in (21),

$$
r(k+1)=\underbrace{e_{n}(k+1)}_{\mathrm{I}}+\underbrace{\lambda_{1} e_{n-1}(k+1)}_{\mathrm{II}}+\cdots+\underbrace{\lambda_{n-1} e_{1}(k+1)}_{\mathrm{III}},
$$


(I) $e_{n}(k+1)=x_{n}(k+1)-y_{d}(k+n)=f(x)+g(x) u(k)+$ $d(k)-y_{d}(k+n)$,

(II) $\lambda_{1} e_{n-1}(k+1)=\lambda_{1}[\underbrace{x_{n-1}(k+1)}_{x_{n}(k)}-y_{d}(k+n-1)]=$ $\lambda_{1} e_{n}(k)$

(III) $\lambda_{p-1} e_{1}(k+1)=\lambda_{p-1}[\underbrace{x_{1}(k+1)}_{x_{2}(k)}-y_{d}(k-1)]=$ $\lambda_{p-1} e_{2}(k)$

Thus, by substituting (I), (II), and (III) in the above equation,

$$
\begin{aligned}
r(k+1)= & f(x)+g(x) u(k)+d(k)-y_{d}(k+P) \\
& +\lambda_{1} e_{P}(k)+\cdots+\lambda_{P-1} e_{2}(k) .
\end{aligned}
$$

Equation (25) can be rewritten as follows:

$$
v(k)=\tilde{g}(k) u_{c}(k)+\tilde{f}(k) .
$$

By using (23), (25), and (B.2),

$$
\begin{aligned}
r(k+1)= & v(k)-v(k)+f(x)+g(x) u(k)+d(k) \\
& -y_{d}(k+P)+\lambda_{1} e_{P}(k)+\cdots+\lambda_{P-1} e_{2}(k) \\
= & k_{v} r(k)-v(k)+f(x)+g(x)+d(k) \\
= & k_{v} r(k)+(f(x)-\tilde{f})+\left(g(x) u-\widetilde{g} u_{c}\right)+d(k) .
\end{aligned}
$$

Also by considering the above equation, the tracking errors, $\widetilde{u}_{f}$ and $\tilde{u}_{g}$, are acquired as follows:

$$
\begin{aligned}
r(k+1)= & k_{v} r(k)+(f(x)-\widetilde{f})+\left(g(x) u_{c}-\widetilde{g} u_{c}\right) \\
& +d(k)+\left(g u-g u_{c}\right) \\
= & k_{v} r(k)+(f(x)-\widetilde{f})+\left(g(x) u_{c}-\tilde{g} u_{c}\right) \\
& +d(k)+g u_{d},
\end{aligned}
$$

where $u_{d}=u-u_{c}$. By the following definitions about the $g(x)$, $\tilde{f}, f(x)$, and $\tilde{g},($ B.5) is rewritten as follows:

$$
\begin{gathered}
f(x)=u_{f}^{T}+\varepsilon_{f}(x) \Longrightarrow \tilde{f}=\widehat{u}_{f}^{T}, \\
g(x)=u_{g}^{T}+\varepsilon_{g}(x) \Longrightarrow \widetilde{g}=\widehat{u}_{g}^{T}, \\
\tilde{u}_{f}=u_{f}-\widehat{u}_{f}, \quad \widetilde{u}_{g}=u_{g}-\widehat{u}_{g}, \\
r(k+1)=k_{v} r(k)+\left(u_{f}^{T}-\widehat{u}_{f}^{T}\right)+\left(u_{g}^{T} u_{c}-\widehat{u}_{g}^{T} u_{c}\right) \\
+\varepsilon_{f}+\varepsilon_{g} u+d(k)+g u_{d} \\
=k_{v} r(k)+\tilde{u}_{f}^{T}+\tilde{u}_{g}^{T} u_{c}+\varepsilon_{f}+\varepsilon_{g} u+g u_{d}
\end{gathered}
$$

with substitution of $\varepsilon^{\prime}=\varepsilon_{f}+\varepsilon_{g} u_{c}+d$ in (B.6); final relation of tracking error is demonstrated as follows:

$$
r(k+1)=k_{v} r(k)+\widetilde{u}_{f}^{T}+\widetilde{u}_{g}^{T} u_{c}+\varepsilon^{\prime}+g u_{d} .
$$

Remark B.1. In consideration of (20) in this region, the following equalities are given:

$$
\begin{aligned}
\tilde{u}_{f}= & u_{f}-\widehat{u}_{f} \Longrightarrow \widetilde{u}_{f}(k+1)=(1-\alpha) \tilde{u}_{f}(k) \\
& -\alpha\left(k_{v} r(k)+\tilde{u}_{g}^{T} u_{c}+\varepsilon^{\prime}+g u_{d}\right)^{T}, \\
\tilde{u}_{g}= & u_{g}-\widehat{u}_{g} \Longrightarrow \widetilde{u}_{g}(k+1)=(1-\beta) \tilde{u}_{g}^{T}(k) u_{c} \\
& -\beta\left(k_{v} r(k)+\tilde{u}_{f}^{T}+\varepsilon^{\prime}+g u_{d}\right)^{T} .
\end{aligned}
$$

By using equation (B.4) in the second region, tracking errors, $\tilde{u}_{f}, \widetilde{u}_{g}$, are obtained as follows:

$$
\begin{aligned}
r(k+1) & =k_{v} r(k)+(f(x)-\tilde{f})+\left(g(x) u-\tilde{g} u_{c}\right)+d(k) \\
& =k_{v} r(k)+(f(x)-\tilde{f})+\bar{g}_{d}+d(k) .
\end{aligned}
$$

Remark B.2. By utilizing (20) in this region, $\tilde{u}_{f}$ and $\tilde{u}_{g}$ are acquired as follows:

$$
\begin{gathered}
\tilde{u}_{f}=u_{f}-\widehat{u}_{f}, \\
\tilde{u}_{f}(k+1)=(1-\alpha) \tilde{u}_{f}(k)-\alpha\left(k_{v} r(k)+\overline{g u}_{d}+\varepsilon^{\prime}\right)^{T} \\
\tilde{u}_{g}=u_{g}-\widehat{u}_{g} \Longrightarrow \tilde{u}_{g}(k+1)=\tilde{u}_{g}(k) .
\end{gathered}
$$

\section{References}

[1] M. Yan, C. Xue, and X. Xie, "A novel model free adaptive controller with tracking differentiator," in Proceedings of the IEEE International Conference on Mechatronics and Automation (ICMA '09), pp. 4191-4196, Changchun, China, August 2009.

[2] A. Xu, Y. Zheng, Y. Song, and M. Liu, "An improved model free adaptive control algorithm," in Proceedings of the 5th International Conference on Natural Computation (ICNC '09), pp. 70-74, Tianjian, China, August 2009.

[3] Q. Gao, D. Ren, C. Dong, and Z. Jia, “The Study of Model-Free Adaptive Controller based on dSPACE," in Proceedings of the 2nd International Symposium on Intelligent Information Technology Application, pp. 608-611, Shanghai, China, December 2008.

[4] H. Zhongsheng and J. Shangtai, "A novel data-driven control approach for a class of discrete-time nonlinear systems," IEEE Transactions on Control Systems Technology, vol. 19, no. 6, pp. 1549-1558, 2011.

[5] S. Jagannathan, "Adaptive fuzzy logic control of feedback linearizable discrete-time nonlinear systems," in Proceedings of the IEEE International Symposium on Intelligent Control Dearborn, September 1996.

[6] S. G. Cheng, "Model-free adaptive process control," United States Patent, 6055524. Washington: United States Patent and Trademark Office of theUnited States Department of Commerce, 2000. 
[7] R. Ordóñez, J. Zumberge, J. T. Spooner, and K. M. Passino, "Adaptive fuzzy control: experiments and comparative analyses," IEEE Transactions on Fuzzy Systems, vol. 5, no. 2, pp. 167-188, 1997.

[8] S. Jagannathan, "Adaptive fuzzy logic control of feedback linearizable discrete-time dynamical systems under persistence of excitation," Automatica, vol. 34, no. 11, pp. 1295-1310, 1998.

[9] P. K. Sinha and A. N. Pechev, "Nonlinear $\mathrm{H}^{\infty}$ controllers for electromagnetic suspension systems," IEEE Transactions on Automatic Control, vol. 49, no. 4, pp. 563-568, 2004. 


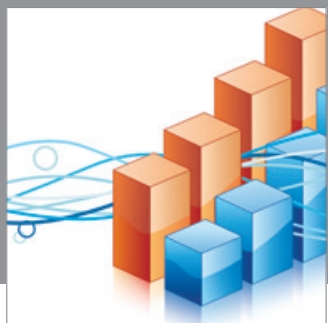

Advances in

Operations Research

mansans

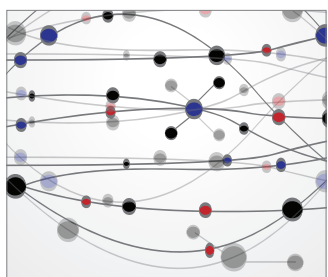

The Scientific World Journal
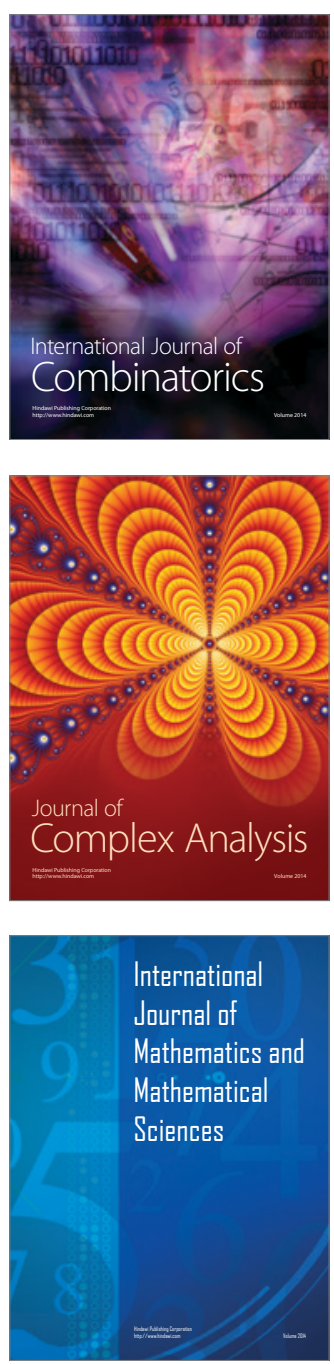
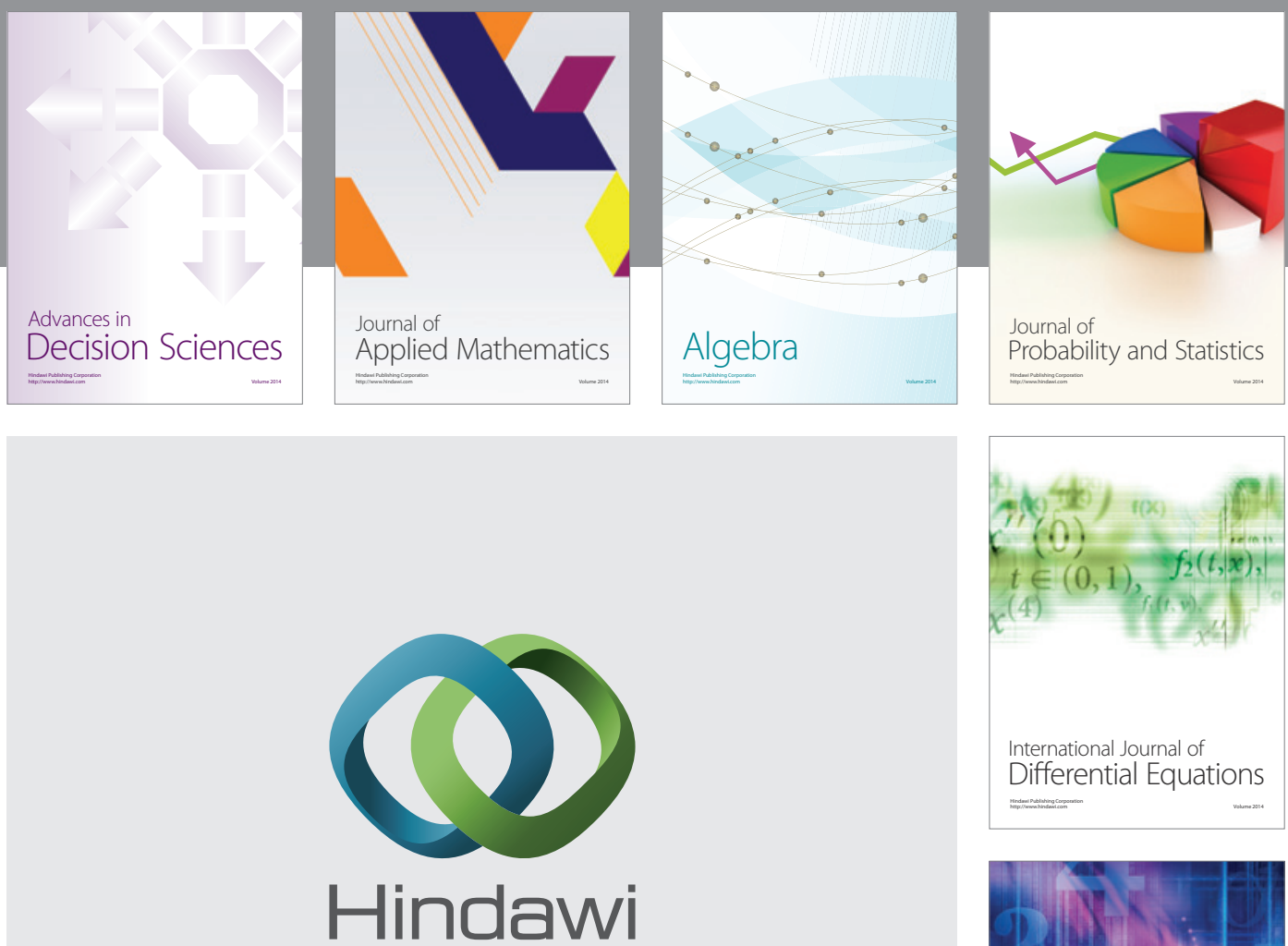

Submit your manuscripts at http://www.hindawi.com
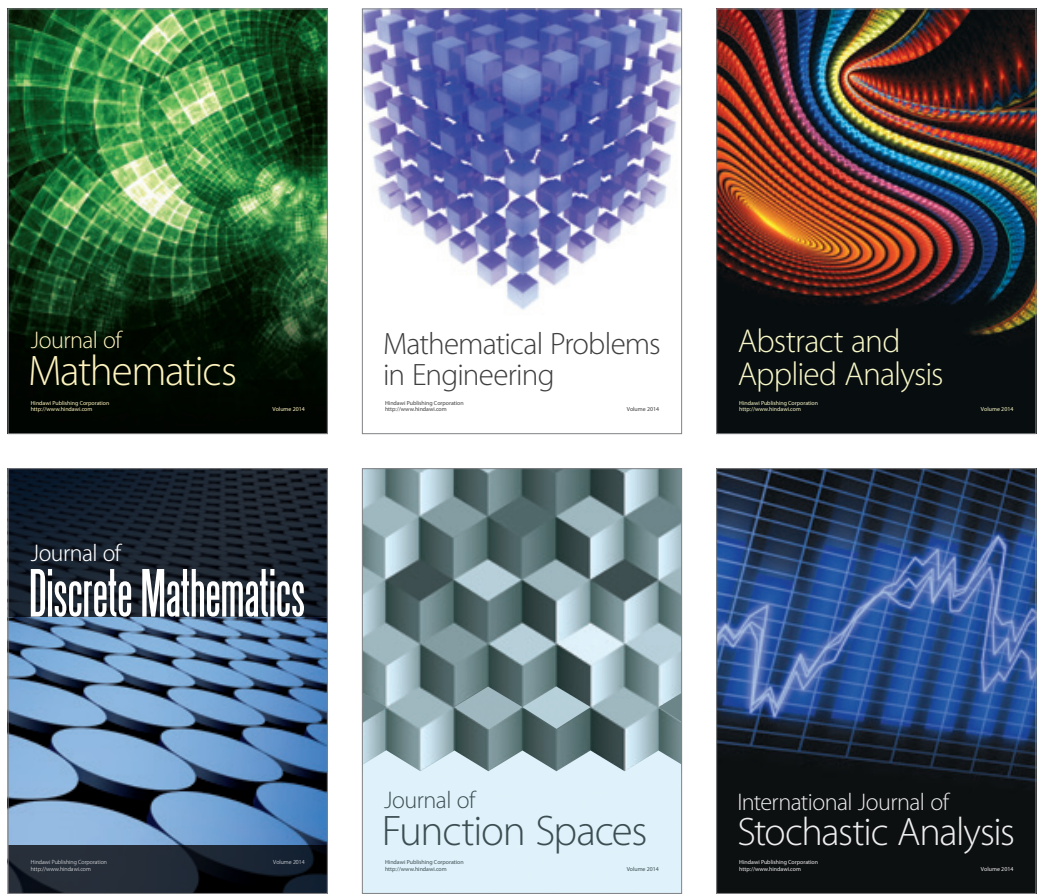

Journal of

Function Spaces

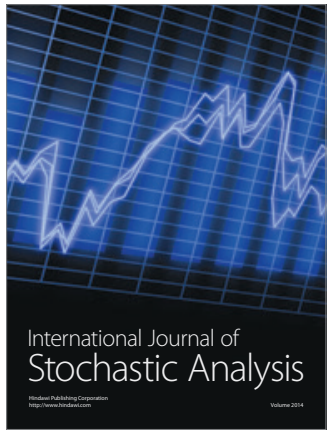

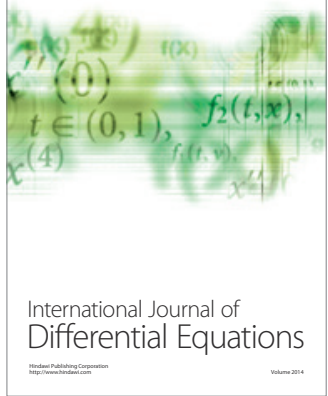
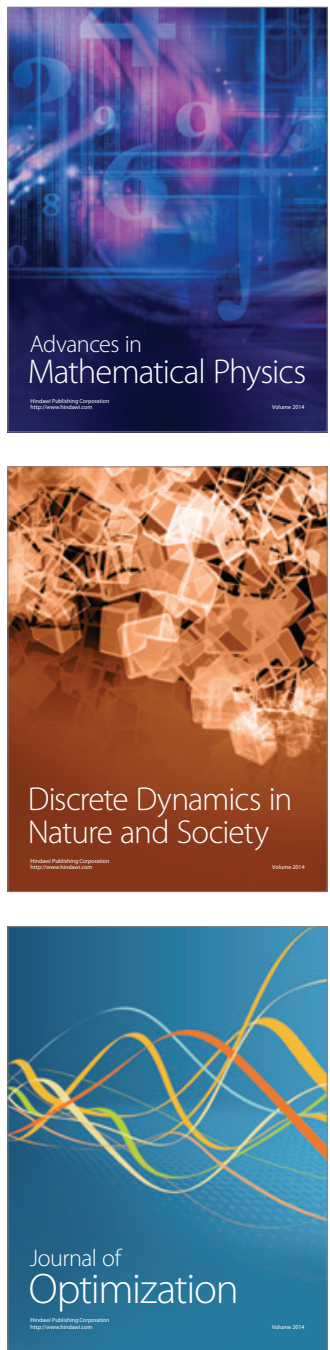